\title{
A presença do genitor no pré-natal: um estudo de representações sociais com gestantes
}

\author{
Fathers' presence in prenatal care: study of social representations among pregnant women \\ La presencia del progenitor en el prenatal: un estudio de representaciones sociales con embarazadas
}

\author{
Pablo Luiz Santos Couto'; Antônio Marcos Tosoli Gomes"; Alba Benemérita Alves Vilela"l'; \\ Samantha Souza da Costa Pereira ${ }^{\text {iv }}$; Luiz Carlos Moraes Françav ; Virgínia Paiva Figueiredo Nogueira ${ }^{\text {VI }}$
}

\begin{abstract}
RESUMO
Objetivo: analisar as representações sociais da presença do genitor no pré-natal para as mulheres gestantes. Método: estudo descritivo e qualitativo, fundamentado na Teoria da Representações Sociais. Contribuíram com o estudo 28 gestantes que realizavam as consultas do pré-natal e responderam a um roteiro de entrevista em profundidade contendo três questões abertas, cujas respostas foram submetidas à Análise de Conteúdo Lexical, possibilitada pelo software IRAMUTEQ. Resultados: a análise aponta a palavra "não" como a mais latente no sistema cognitivo das gestantes, sendo percebida a alta frequência no Dendograma de Classes, presença no eixo de intersecção entre as ordenadas e abscissas no Mapa Fatorial de Correspondência, além de ser central e fazer as maiores forças de conexidade com as demais palavras na árvore máxima de similitude. Conclusão: as representações sociais das gestantes sobre a presença do genitor durante as consultas de pré-natal foram elaboradas a partir da negação, evidenciadas nos discursos do grupo no termo "não".
\end{abstract}

Descritores: Enfermagem; Obstetrícia; Gestantes; Cuidado Pré-Natal; Relações Pai-Filho.

\begin{abstract}
Objective: to examine social representations held by pregnant women of the fathers' presence in prenatal care. Method: this qualitative, descriptive study, based on Social Representations Theory, involved 28 pregnant women undergoing prenatal consultations, who answered an in-depth, scripted interview containing three open questions. Their responses were analyzed using Lexical Content Analysis, made possible by IRAMUTEQ software. Results: the analysis pointed to the word "no" as the most latent in the pregnant women's cognitive system: it was found at high frequency in the Dendrogram of Classes, was present at the axis of intersection between ordinates and abscissas on the Factorial Correspondence Map, besides being central and showing strongest connectedness with the other words in the similarity tree. Conclusion: the pregnant women's social representations of the fathers' presence at prenatal appointments were elaborated on the basis of denial, evidenced in the group's discourse in the term "no".
\end{abstract}

Descriptors: Nursing; Obstetrics; Pregnant Women; Prenatal Care; Father-Child Relations.

\section{RESUMEN}

Objetivo: analizar las representaciones sociales que tienen las mujeres embarazadas sobre la presencia del padre en la atención prenatal. Método: este estudio cualitativo, descriptivo, basado en la Teoría de las Representaciones Sociales, involucró a 28 gestantes en consulta prenatal, quienes respondieron una entrevista en profundidad y guionizada que contenía tres preguntas abiertas. Sus respuestas se analizaron mediante el análisis de contenido léxico, posible gracias al software IRAMUTEQ. Resultados: el análisis apuntó a la palabra "no" como la más latente en el sistema cognitivo de la gestante: se encontró con alta frecuencia en el Dendrograma de Clases, estuvo presente en el eje de intersección entre ordenadas y abscisas en el Mapa de Correspondencia Factorial, además de ser central y mostrar una conexión más fuerte con las otras palabras en el árbol de similitudes. Conclusión: las representaciones sociales de las mujeres embarazadas sobre la presencia de los padres en las citas prenatales se elaboraron sobre la base de la negación, evidenciada en el discurso del grupo en el término "no". Descriptores: Enfermería; Obstetricia; Mujeres Embarazadas; Atención Prenatal; Relaciones Padre-Hijo.

\section{INTRODUÇÃO}

O acompanhamento pré-natal realizado por profissionais de saúde durante a gestação é fundamental para o desenvolvimento saudável do feto e o controle das condições clínicas das gestantes ${ }^{1-2}$.

'Enfermeiro. Mestre. Professor Auxiliar. Centro de Ensino Superior de Guanambi. Guanambi, Bahia, Brasil. E-mail: pablocouto0710@yahoo.com.br. ORCID: https://orcid.org/0000-0002-2692-9243. "Enfermeiro. Doutor. Professor Adjunto. Universidade do Estado do Rio de Janeiro. Rio de Janeiro, Brasil. E-mail: mtosoli@gmail.com. ORCID: https://orcid.org/0000-0003-4235-9647 "'Enfermeira. Doutora. Professora Plena. Universidade Estadual do Sudoeste da Bahia. Jequié, Brasil: albavilela@gmail.com. ORCID: https://orcid.org/0000-0002-1187-0437 IVEnfermeira. Mestra. Professora Auxiliar. Centro de Ensino Superior de Guanambi. Guanambi, Brasil. E-mail: samantha.uefs@gmail.com. ORCID: https://orcid.org/0000-0002-1533-4017 VEnfermeiro. Mestre. Doutorando. Universidade do Estado do Rio de Janeiro. Rio de Janeiro, Brasil. E-mail: Icmoraesfranca@hotmail.com. ORCID: https://orcid.org/00000002-6370-115X.

VIEnfermeira. Mestre. Doutoranda. Universidade do Estado do Rio de Janeiro. Rio de Janeiro, Brasil. E-mail: virginiafigueiredo@yahoo.com.br. ORICD: https://orcid.org/0000-0001-7331-9715. 
Na fase gestacional a mãe está sendo preparada fisiologicamente e psicologicamente para a chegada do concepto, já que a ela são atribuídos os cuidados com este novo e frágil ser, em decorrência da construção social e cultural da maternidade ${ }^{3}$. Nessa perspectiva, grande parte delas, ao acessar o serviço de saúde, está sozinha ou na companhia da mãe ou uma amiga ${ }^{4-5}$, normalmente figuras do sexo feminino.

Percebe-se, então, tendência a pouca ou nenhuma contribuição ou participação do pai ou companheiro durante esta fase experenciada pela mulher, em que o homem tem assumido, como perfil histórico-social, um papel secundário ${ }^{6}$. Contudo, há homens que também não se interessam por esse momento da mulher e a preparação para a chegada do/a filho/a, seja por reafirmar a construção de gênero sobre a maternidade e não querer participar das consultas, seja porque muitas delas não concordam com a presença deles nas consultas ${ }^{3}$.

Algumas pesquisas evidenciam que a presença desses homens pode favorecer o suporte emocional à companheira, quando esta concorda com sua presença, entendendo que as reponsabilidades com o filho são de ambos e devem ser divididas. Entretanto, estudos também revelara a negação da presença do genitor, às vezes pela representação do medo que muitas possuem dos seus companheiros e este momento com o profissional ser apreendido como libertador ${ }^{5-7}$.

Faz-se necessário que todos/as profissionais responsáveis pelo pré-natal dialoguem com as gestantes sobre estas questões, sendo como importante incluir os futuros pais, visando à saúde e o desenvolvimento do/a filho/a. Ao mesmo tempo, a ausência deles pode indicar questões subjetivas que podem interferir na saúde materno-fetal, como a violência doméstica de gênero, muito comum nesses $\operatorname{casos}^{5-6}$.

Destarte, este estudo justifica-se pela possibilidade de ampliar a compreensão em que estão fundamentadas as representações sociais de gestantes sobre a presença do genitor durante as consultas pré-natal, uma vez que estudos têm considerado que a presença do pai durante o período gravídico pode contribuir para uma qualidade de vida materno-fetal. Por esse motivo, objetiva-se analisar as representações sociais da presença do genitor no pré-natal para as mulheres gestantes.

\section{REFERENCIAL TEÓRICO}

A Teoria das Representações Sociais (TRS) é adequada a este tipo de estudo por possibilitar a idealização de uma realidade comum, que contribui para a comunicação e compartilhamento de informação e formação do grupo de pertencimento ${ }^{8}$. Dessa forma, as representações sociais funcionam como mecanismos que refletem fenômenos sociais produzidos no sistema cognitivo, compreendidos por meio dos significados elaborados na memória ${ }^{9}$.

A TRS, em sua abordagem processual, possui dois mecanismos denominados de objetivação e ancoragem, que possibilitam o entendimento do processo de formação das representações sociais ${ }^{8-9}$. A teoria também permite a investigação da construção social e mental de ideias, significados, comportamentos e práticas de grupos ${ }^{8,10}$.

A abordagem processual da TRS favorece o modo sobre como se processam e se constroem os significados relacionadas à realidade comum a um grupo social - grupo de pertencimento - que compartilha um conjunto de conceitos, proposições e vivências interpessoais cotidianas, constituindo-se em teoria do senso comum ${ }^{9-11}$.

\section{MÉTOdo}

Estudo descritivo e qualitativo, baseado na perspectiva processual da Teoria das Representações Sociais.

Procedeu-se com a coleta de informações em abril de 2017 em duas Estratégias Saúde da Família da Atenção Primária na cidade de Guanambi, Bahia (BA), após as consultas de pré-natal realizadas pelas enfermeiras. A priori, foram convidadas 30 gestantes que realizaram consultas em serviços da Atenção Básica do município, que atenderam aos critérios de inclusão: maiores de 18 anos e cadastrado no SISPRENATAL. Contudo, duas foram excluídas por declararem, durante as entrevistas, não possuir condições emocionais e, assim, impossibilitadas de interagir por comunicação verbal com o pesquisador. Referiram lembrar-se de algum fator traumático que não gostariam de compartilhar.

Utilizou-se um roteiro de entrevista em profundidade, com três questões abertas, para obter as informações junto às participantes: "Fale-me da importância das consultas do pré-natal durante a gestação da senhora", "Fale-me o que senhora pensa sobre a presença de seu parceiro (pai da criança) nas consultas de pré-natal" e "Caso a senhora queira a presença dele, fale-me como a enfermeira pode contribuir para que o pai ou o acompanhante/parceiro participe das consultas pré-natal". Para validação dos resultados, foi utilizada a saturação empírica, quando há uma saturação teórica dos discursos, ou seja, presencia-se uma repetição no conteúdo das falas e, neste momento, torna-se desnecessária a inclusão de mais participantes.

Os discursos das participantes foram gravados pelos pesquisadores em um gravador MP3 Player. Em seguida, foram transcritas e organizadas na íntegra no software Microsoft Word 2016. Por fim, submetidas à Análise de Conteúdo Lexical, possibilitada pelo software Interface de R Pour Les Analyses Multidimnsionnelles de Textes et Questionnaires 
(IRAMUTEQ), o qual emitiu três gráficos e suas respectivas análises, a saber: o Dendrograma de Classes para a Classificação Hierárquica Descendente, o Mapa Fatorial para Análise Fatorial de Correspondência (AFC) e a Árvore Máxima de Similitude, para a análise de similitude ${ }^{12}$.

O Dendograma de Classes, a partir da análise do conteúdo e das funções dos vocábulos nas frases que fazem parte dos discursos, mostra quais palavras foram importantes em seus contextos e obtiveram maiores qui-quadrado $\left(x^{2}\right)$ e frequências em suas correlações com as características dos/as participantes, permitindo a Classificação Hierárquica Descendente (CHD). Ressalta-se que as principais frequências poderão ser as palavras que favorecem a formação representacional $^{13}$, cuja confirmação é ressaltada no Mapa Fatorial de Correspondência, com a análise fatorial dos termos que se aproximam do eixo central.

A AFC decorrente do mapa permite, por meio de correlações entre as variáveis sociodemográficas e os léxicos com maiores frequências e coocorrências presentes nos discursos, identificar congruências e divergências dentro do grupo de pertencimento, e, assim, delimitar as palavras com maior $x^{2}$, que contribuem para o eixo zero (0) do gráfico das ordenadas e abscissas ${ }^{14}$.

A árvore máxima de similitude apresenta a coocorrência dos termos e o índice de similitude das palavras (duas a duas) que compuseram o Mapa Fatorial e o Dendograma de Classes, quando se consideraram apenas os participantes que apresentaram em seus discursos, ao menos, duas palavras importantes para a elaboração dos significados representacionais, estruturados nos campos cognitivos, pois a relação de conexidade existe apenas entre um e outro termo ${ }^{14-16}$, os quais contribuem para entender em quais processos se ancoram as representações, a partir do sentido que, as palavras juntas possuem.

Ressalta-se que, antes da aplicação do instrumento de coleta, foi apresentado o Termo de Consentimento Livre e Esclarecido às participantes e, logo após a leitura, solicitou-se a sua assinatura, obedecendo à Resolução 466/2012, sobre pesquisas envolvendo seres humanos. O projeto foi aprovado pelo Comitê de Ética e Pesquisa da instituição, com o parecer número 2022888/2017.

\section{RESULTADOS}

Os dados decorrentes das entrevistas e processados no software IRAMUTEQ, a partir da análise estatística padrão, originou um corpus constituído de 28 unidades de contexto iniciais (UCI), totalizando 4054 ocorrências, 885 palavras distintas e média de 34 ocorrências por palavra, com frequência igual ou superior à média e com $x^{2} \geq 3,206$. Após reduzir o vocabulário às suas raízes lexicais, foram encontrados 584 radicais reduzidos e analisáveis, sendo 507 unidades de contextos elementares (UCE). A Classificação Hierárquica Descendente (CHD) reteve $88,24 \%$ do total das UCE do corpus, que foram organizadas em três classes, como pode ser visualizado na Figura 1.

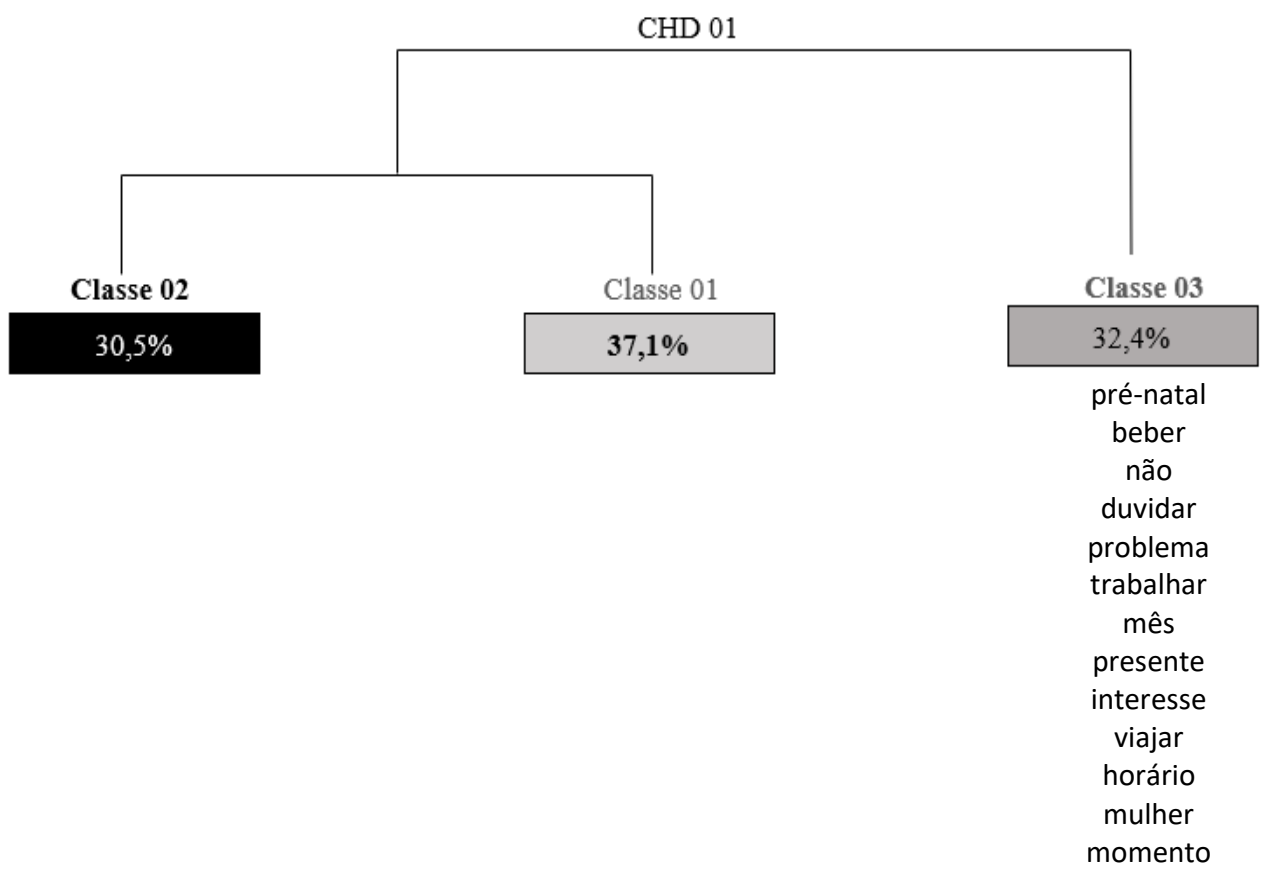

FIGURA 1: Dendograma de Classes para Classificação Hierárquica Descendente. Guanambi-BA, 2018. 
Percebe-se no Dendograma de Classes que o corpus inicial sofreu uma divisão originando dois agrupamentos, o da esquerda aglutinando as classes 1 e 2 e o da direita que agrupou a classe 3 . A classe 1, evidenciou 39 UCE, com 105 palavras analisáveis, significando 37,1\% do corpus, a classe 2, envolveu 32 UCE, contendo 105 palavras analisáveis com um total de 30,5\% e a 3 apresentou 34 UCE, 105 palavras, contabilizou 32,4\% do corpus.

A seguir será aprofundada a classe 3, importante para o entendimento do objeto de investigação deste presente estudo, que evidencia os vocábulos com maior $\mathrm{x}^{2}$ e as variáveis-atributos que contribuíram significativamente. Essa última classe comporta o vocábulo 'não' com $x^{2}=19,01$ e, ainda que não seja o primeiro a compor a classe, foi o que apresentou alta frequência de repetição e com mais conexões entre as demais palavras, reforçando a análise de que a negativa da participação está presente no sistema representacional das participantes, sendo ancorada na ideia e no sentimento de negação quanto à presença do genitor nas consultas.

A AFC favorecida pelo mapa/plano fatorial apresentada na Figura 2, outra técnica de análise emitida pelo IRAMUTEQ, corrobora e reforça os achados no Dendograma para a CHD. Evidenciou-se que a variância total das palavras foi explicada com a soma dos valores percentuais das correlações emergidas com o processamento dos dados, com um total de $100 \%$ de aproveitamento das evocações utilizadas, o que demonstra fidedignidade dos parâmetros estatísticos e consistência das respostas e consenso entre o grupo de pertença, possibilitando uma análise significante. Assim como no Dendograma, considerou-se a frequência mínima de dez palavras, em decorrência da pluralidade do campo semântico elaborado pelas participantes. Foram verbalizadas no total geral das entrevistas 4054 palavras, sendo 885 diferentes.

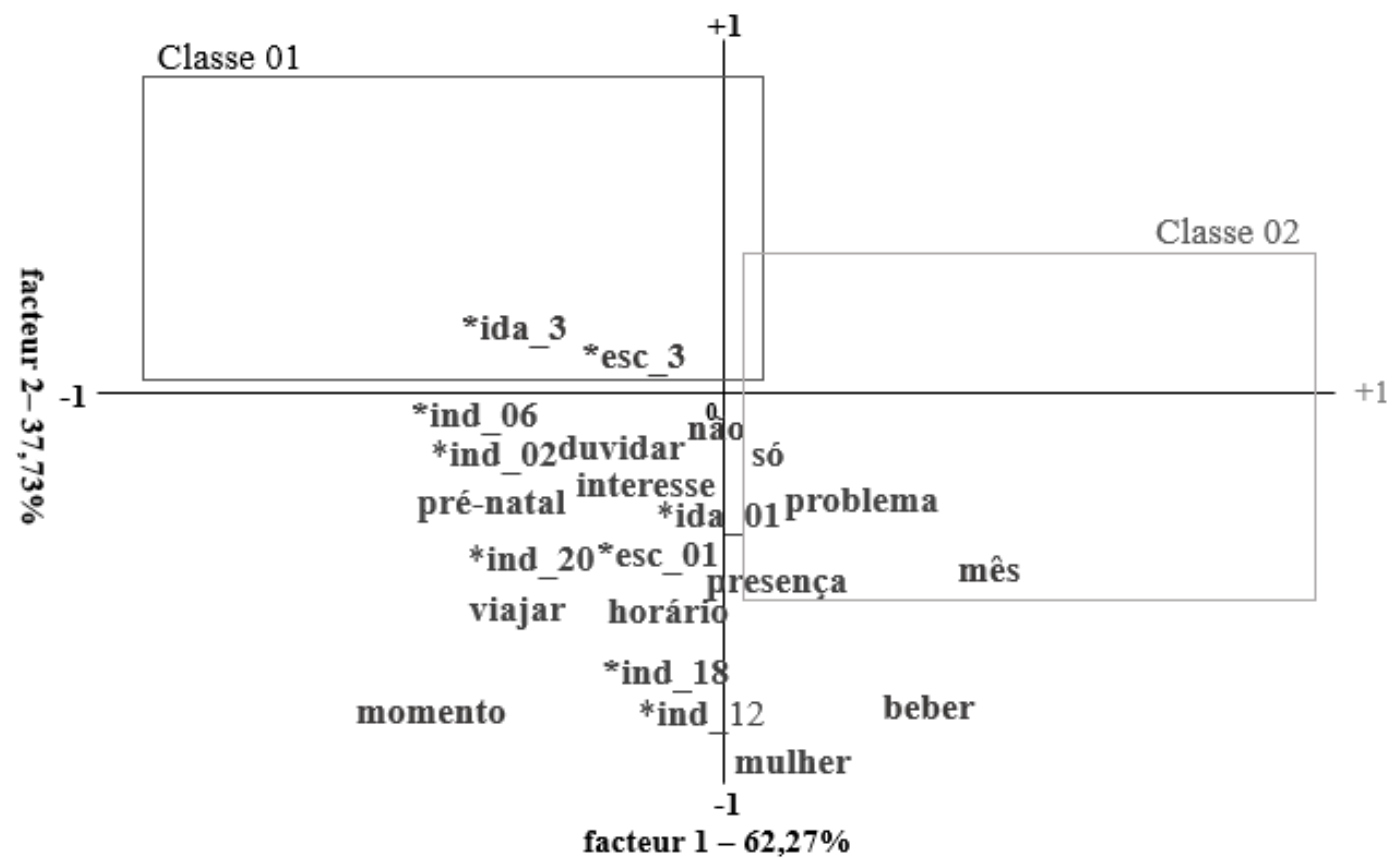

FIGURA 2: Mapa fatorial de Correspondência para Análise Fatorial de Correspondência. Guanambi-BA, 2018.

Salienta-se que as variáveis fixas (identificação sociodemográfica) adotadas para a associação com as variáveis de opinião (as falas decorrentes da entrevista que resultaram nos léxicos/vocábulos) foram: nível de escolaridade (fundamental, médio e superior) e faixa etária (de 18 a 24 anos; 25 a 35 anos e acima dos 35 anos). Desse modo, foi possível proceder com a AFC, percebida nas correlações estabelecidas com as variáveis qualitativas no mapa fatorial de correspondência, portanto, a AFC apresentou variações da organização espacial das variáveis, expondo aproximações e afastamento das variáveis fixas e de opinião nos dois fatores: fator 1 (F1) e fator 2 (F2). Ou seja, expôs a proximidade e a distância entre as características das gestantes e suas respostas. No eixo das abscissas (F1), há tradução das palavras mais significativas, necessárias para a formação das representações, demonstrando $62,27 \%$ da variância total de respostas. Assim como no eixo das ordenadas (F2), ainda que se verifique menor variância, com 37,73\% de contribuição dos vocábulos para o fator, elas também contribuem para o processo de elaboração das representações. 
Ressalta-se que o grupo lexical/vocábulos mais próximo do eixo de intersecção zero ('0') e das linhas que formam as ordenadas e abscissas, tem maior contribuição para o processo de formação das representações sociais, uma vez que delimitam o entendimento sobre em quais significados e ideias são elaborados os conteúdos representacionais. Nesse estudo, assim como no dendograma, considera-se a apresentação espacial da classe 3 e sua influência no mapa fatorial, possibilitando evidenciar sobre em quais experiências estão ancoradas as representações sociais das gestantes sobre a presença do genitor na consulta pré-natal, por meio das palavras com significância do dendograma, uma vez que revelam como são construídos e processados os discursos representacionais.

O mapa fatorial, por meio da correspondência elaborada entre as variáveis, delimita os conjuntos de vocábulos que contribuem para o processo de elaboração das representações sociais das gestantes desse grupo, de modo a apresentar a configuração espacial das três classes emitidas pelo IRAMUTEQ pelo Dendograma de Classes e que, juntas, revelam o que é comum entre as gestantes (palavras próximas ao eixo zero) e o que é destoante. Ou seja, todas as palavras afastadas da zona de interseç̧ão e espalhadas espacialmente no plano fatorial. Contudo, percebe-se que no centro do gráfico, onde os eixos se conectam, é o local de ligação entre as classes e onde se localizam as palavras mais latentes nos sistemas de cognição das participantes.

Por isso, os termos que se apresentaram na classe 3 têm contribuição com as demais classes e, sobretudo, com o eixo central do mapa fatorial, pois conformam um aglomerado de palavras muito próximas ao ponto zero (0) e, assim, contribuem estatisticamente para o processo de formação das representações sociais e em quais aspectos e ideias elas estão ancoradas no que concerne à negação para a presença do pai nas consultas de pré-natal.

As variáveis fixas que apresentaram maior contribuição para os fatores que sofrem influência pela Classe 3 foram: as gestantes com maior grau de escolaridade e acesso ao nível superior (*esc_3), as com idade acima dos 35 anos (*ida_3 corresponde às gestantes com tal faixa etária) no F1+ e F2 -, além das gestantes de número 2 e 6 (correspondente, respectivamente aos códigos: *ind_02, *ind_06); o F1- teve contribuição das gestantes com nível fundamental de escolaridade (*esc_1), aquelas que possuíam idade de 18 a 24 nos dias da coleta (*ida_01) e as gestantes com o código 12, 18 e 20 (respectivamente, *ind_12, *ind_18 e *ind_20). O fator F2 + não teve interferência de nenhuma variável que influenciou na classe 03.

O que torna esse grupo de pertença homogêneo, mesmo com nuances percebidas com as contribuições de evocações nos dois fatores, são os léxicos 'não', 'duvidar' e 'só', pois estão próximos do eixo zero. Outras palavras associadas com maior frequência e maior contribuição estatística para os eixos, segundo ordem de importância pela proximidade com o eixo zero, em concomitância foram: 'interesse', 'problema', 'viajar', 'mulher', 'horário' e 'duvidar'. Mais afastadas dos eixos, mas que dão significados representacionais aos discursos foram 'mês', 'momento' e 'beber'.

Tais evidências podem ser corroboradas com a análise de similitude apresentada na árvore máxima de similitude (Figura 3), que apresenta de modo espacial as evocações com maior contribuição representacional, por terem maior grau e força na conexão entre os vocábulos. Essa apresentação gráfica possibilita perceber como os termos são concatenados por meio da análise prototípica das palavras e como se revelam os sentidos com a conexão entre as mesmas, evidenciando a ancoragem e o caráter multifacetado das representações elaboradas.

Desse modo, as palavras com influência significativa no mapa fatorial são as que possuem as maiores forças de conectividade com o eixo central da possível representação das gestantes, percebida no vocábulo 'não'. Este vocábulo é central na árvore e tem o maior grau de ligação com o termo 'consulta', apresentando com maior clareza o que está latente no sistema de cognição das participantes do estudo, conforme perceptível nos discursos abaixo:

Ele nunca vem, mas eu não gostaria, pois, o momento da consulta com a enfermeira é o momento que a gente tem para conversar, se ele estivesse comigo eu não iria me sentir à vontade [G05]

Eu estou me separando, ele é muito agressivo. No início da gravidez quando ia na médica ele tinha ciúmes, ele não queria ir, e quando bebia implicava mais ainda e achava que eu mentia, insistia que o filho não era dele (...) Hoje ele sabe que o filho é dele, mas não quer ajudar nada, nem ele e nem a família dele, mas eu estou bem, tenho apoio da minha mãe [G07].

Acho muito importante em algumas consultas apenas, pois as consultas nos mantem informados sobre os cuidados desde a gestação até o parto. Mas não quero a presença em todas, já que quero ter conversas a sós com a médica ou a enfermeira [G13].

Pra você ter ideia, no momento da gravidez foi um susto, porque estávamos em processo de separação, brigamos muito ele chegou a me agredir, inclusive já me bateu, e eu estando grávida, achando que minha filha era de outro homem. Eu ainda tentei reatar por conta da bebê. Mas vi que não dava certo. Ele gosta de beber, xinga muito, fala que a filha não é dele, ameaça quando liga. É um horror [G18].

(...) não me sinto mal se não der pra ele estar presente, gosto de estar sozinha [G21]. 


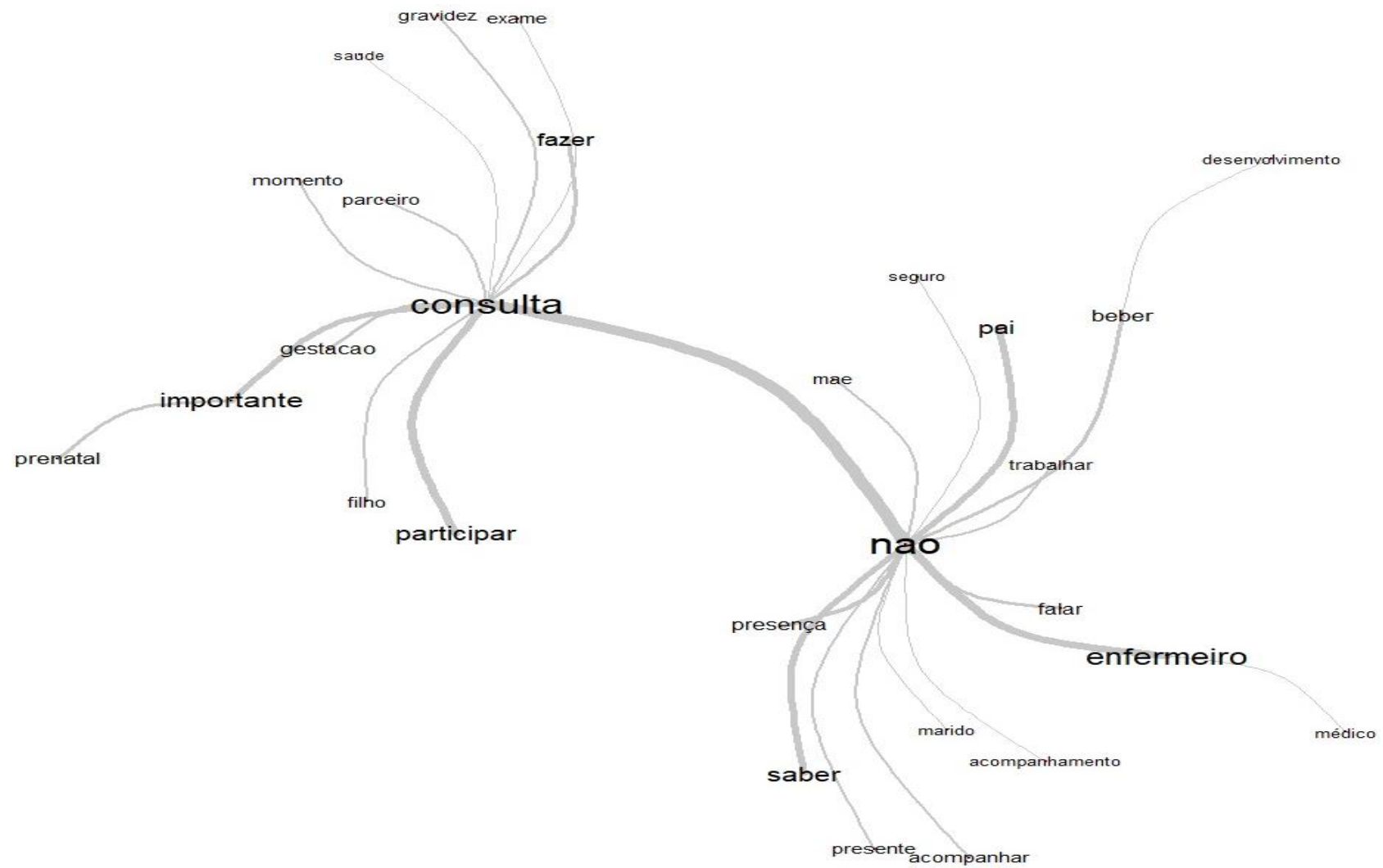

FIGURA 3: Árvore Máxima de Similitude para Análise de Similitude. Guanambi-BA, 2018.

As duas palavras centrais são os principais léxicos que contribuem para esse processo de formação das representações sociais, cujo sentido é entendido quando tais palavras se conectam e ganham significados, possibilitando a ancoragem.

\section{DISCUSSÃO}

Os resultados apontam que as representações sociais do grupo de gestantes sobre a presença do genitor nas consultas pré-natal são elaboradas a partir da negação, cuja ancoragem pode ser explicada tanto por elas não se sentirem bem com a presença dos seus companheiros, o que causa intimidação e medo de se expressarem com a enfermeira, ou porque muitos deles não querem acompanha-las ${ }^{17}$. A mulher, para se defender dessa situação, acaba excluindo a presença paterna das consultas, ficando dessa forma mais segura para expressar seus sentimentos e dúvidas ${ }^{18}$.

Há em alguns momentos, no grupo de gestantes, o desejo da não presença do marido, ainda que não tenham deixado claro se isso ocorria por sofrerem violência doméstica. O silêncio percebido durante as entrevistas e a negação podem apontar para a vivência deste fenômeno em seus lares ${ }^{19}$. Tanto na Etiópia quanto no Vietnã, evidenciou-se que a maioria das mulheres pobres e com baixo nível de escolaridade havia sido espancada, inclusive na região do abdome, pelo pai biológico do bebê e, por isso, demonstravam medo diante de sua presença ${ }^{20-21}$. Tal evidência também denota que as mulheres mais vulneráveis socialmente são as que mais estão expostas a qualquer tipo de violência. $A$ violência de gênero, tende a interferir na saúde mental da mulher não só durante o período gravídico, mas no pós-parto também ${ }^{22}$.

Contudo, não apenas a violência, mas outro fator como a confiança na experiência das mulheres mais velhas com a maternidade, apresentou-se significativamente nas representações das gestantes, o que já fora evidenciado por outras mulheres que não sentiram necessidade da presença dos companheiros, mas de suas mães durante o pré-natal ${ }^{21,23}$. Dessa 
maneira, as gestantes ancoram suas representações, quanto à presença de suas mães, no apoio familiar de segurança e de estímulo ${ }^{23}$.

A participação do genitor no pré-natal pode ser estimulada durante as atividades de consulta de grupo e serve para preparar o casal para a hora do parto, isso se as mulheres permitirem e aceitarem pois, ao mesmo tempo que é importante para o papel de cuidador, pode ser danoso do ponto de vista da negação delas, por vários motivos, como medo do parceiro e liberdade de estar longe das ameaças de violência ${ }^{20}$.

Alguns outros fatores que ancoraram as representações das gestantes no termo 'não' foram as viagens e/ou trabalho dos genitores, que impossibilita sua presença durante esse momento na vida de sua companheira ${ }^{24,25}$. Além disso, não sendo possível sua liberação para acompanhar as consultas de pré-natal, ele goza da liberação da licença paternidade, de apenas cinco dias, para registrar a criança e ajudar a mulher na adaptação da nova rotina nos primeiros dias pós-parto ${ }^{26-27}$

Atualmente, tem-se discutido sobre a preocupação social da participação do companheiro em todas as fases do ciclo gravídico-puerperal. Contudo, alguns profissionais da saúde não se atentam para a vontade da mulher. Sendo assim, muitos podem, em decorrência da cultura machista e socialmente construída, relegar exclusivamente à mulher o papel da maternidade e o cuidado com os filhos ${ }^{28}$. Dessa forma, muitos homens estão ausentes nessa etapa e sem consciência de seu papel de cuidador ${ }^{4-5,7-8}$.

No que concerne à construção social dos gêneros, os papéis do homem e da mulher são constituídos culturalmente e mudam conforme a sociedade e o tempo, atribuindo à mulher e ao homem funções diferentes no meio social. Desde a sua mais tenra idade, a menina é incentivada pela família, sociedade e religião à prática da maternidade, com objetos infantis e brincadeiras, como cuidar da boneca, brincar de casinha, fazer 'comidinha' ${ }^{24}$. Por sua vez, ao homem é imputado o papel de provedor do lar, o qual tem que trabalhar para sustentar a casa, a esposa e os filhos, com a permissão social de ocupar os espaços públicos, restando a mulher o espaço privado: a maternidade e as tarefas domésticas ${ }^{27,29}$

As representações revelaram que algumas participantes não têm nenhum relacionamento amoroso com o parceiro, e por isso não demonstra interesse quanto à participação dele. Há evidência de que algumas mulheres não queriam mais continuar com o pai da criança, levando-o ao abandono do seu filho, não mostrando preocupação e interesse nas consultas ${ }^{6,18,21}$. Em Cingapura e em outras sociedades asiáticas, por exemplo, estabelecendo um comparativo, as mães solteiras e jovens também são estigmatizadas, sofrem preconceitos decorrentes dos valores tradicionais machistas e patriarcais que desvelam as desigualdades de gênero, o que interfere na busca pelos cuidados pré-natal ${ }^{30}$.

Considera-se limitação do estudo sua realização em um município do nordeste do Brasil, o que impossibilita generalização dos resultados, uma vez que as mulheres têm vivências que variam conforme a cultura e a localidade. Entretanto, sua relevância está no fato de que este é um estudo qualitativo e que aponta subjetividades oriundas de representações sociais que coadunam com aquelas apresentadas em estudos realizados em outros cenários e, outras representações específicas, que revelam as singularidades do grupo de mulheres aqui estudadas. Assim, oferece contribuições para profissionais de saúde, sobretudo as enfermeiras, na medida em que se apontam reflexões que podem reverberar estratégias de cuidado que cooperam com a saúde do materno-infantil.

\section{CONCLUSÃO}

Conclui-se que as representações sociais das gestantes foram elaboradas a partir da negação da presença do genitor nas consultas de pré-natal, pela palavra ' $n a \tilde{o}$ ' evidenciada nos discursos. Tal termo, quando conectado aos demais que também foram relevantes para o processo de elaboração representacional, revela em quais aspectos e ideias estão ancoradas: as mulheres negam a presença do companheiro, por sofrerem violência doméstica e de gênero e sentirem na consulta um momento de libertação, ou simplesmente por quererem estar sozinhas.

Outrossim, as representações elaboradas por elas também podem indicar situações de vulnerabilidade durantes a gestação e as enfermeiras, ao refletir sobre tais questões, poderão focar suas ações de promoção à saúde numa assistência que amenize tais situações e possibilite melhor enfrentamento por parte das gestantes, bem como a denúncia dos agressores.

\section{REFERÊNCIAS}

1. Semente PASN, Macedo VF, Fernandes ERL, Teixeira GA, Araújo MG, Carvalho JBL. Experiences of men in cases of high-risk pregnancy of their partners. J. health biol. sci. [Internet]. 2016 [cited 2019 Mar 14]; 4(3):181-86. DOI: http://dx.doi.org/10.12662/2317-3076jhbs.v4i3.751.p181-186.2016 
2. Tascón LAM, Guatibonza MDA, Ospina CBP, Tascón LIM, Penagos SM, Bahena AMV. Influencia de la adolescencia y su entorno en la adherencia al control prenatal e impacto sobre la prematuridad, bajo peso al nacer y mortalidad neonatal. Rev. chil. obstet. ginecol. [Internet]. 2015 [cited 2019 mar 14]; 80(4):306-31. DOI: http://dx.doi.org/10.4067/S071775262015000400005

3. Ferreira TN, Almeida DR, Brito HM, Cabral JF, Marín HÁ, Campos FMC. The importance of paternal involvement during prenatal care: perception of the mother and father in the city of Caceres - MT. Rev. G\&S [Internet]. 2014 [cited 2019 Mar 14]; c5(2):33745. DOI: https://periodicos.unb.br/index.php/rgs/article/view/432/415

4. Rêgo RMV, Souza AMA, Rocha TNA, Alves MDS. Paternity and breastfeeding: mediation of nurses. Acta paul. enferm. [Internet]. 2016 [cited 2019 mar 14]; 29(4):374-80. DOI: http://dx.doi.org/10.1590/1982-0194201600052

5. Figueiredo MGAV, Marques AC. Fathers' pre-natal experiences. Cogitare enferm. [Internet]. 2011 [cited 2019 Mar 14]; 16(4):708-13. DOI: http://dx.doi.org/10.5380/ce.v16i4.26126

6. Benazzi AST, Lima ABS, Sousa AP. Prenatal care for men: a new look at the man's presence. Rev. pol. Públ. [Internet]. 2011 [cited 2019 Mar 14]; 15(2):327-33. Available from:

http://www.periodicoseletronicos.ufma.br/index.php/rppublica/article/view/849/871

7. Cruz RSBLC, Caminha MFC, Filho MB. Aspectos Históricos, Conceituais e Organizativos do Pré-natal. Rev. bras. cienc. Saúde [Internet]. 2014 [cited 2019 Mar 14]; 18(1):87-94. Available from: http://www.periodicos.ufpb.br/ojs2/index.php/rbcs/article/view/15780/11722

8. Moscovici S. Representações Sociais: Investigações em Psicologia Social. Rio de Janeiro: Vozes; 2015.

9. Jodelet D. Representações sociais: um domínio em expansão. In: JODELET, D. (Org.). As representações sociais. Rio de Janeiro: EdUERJ, 2001.

10. Couto PLS, Paiva MSP, Gomes AMT, Sorte ETB, Rodrigues LSA, Coelho EAC. Meanings of HIV/AIDS prevention and sexuality for young Catholics. Rev. gaúch. enferm [Internet]. 2017 [cited 2019 mar 14]; 38(4): e2016-0080. DOI: https://doi.org/10.1590/1983-1447.2017.04.2016-0080

11. Teixeira MA, Paiva MS, Couto PLS, Oliveira JF, Wolter RMCP. Feelings of seropositive women towards non-breastfeeding. Rev. baiana enferm. [Internet]. 2017 [cited 2019 mar 14]; 31(3):e21880. DOI: http://dx.doi.org/10.18471/rbe.v31i3.21870

12. Russell S, Norvig P. Representação de conhecimento. In: Russell S, Norvig P. Inteligência Artificial. Trad. Simille RC. 3. ed. Rio de Janeiro: Campus; 2015. p. 381-419.

13. Couto PLS, Paiva MS, Oliveira JF, Gomes AMT, Rodrigues LSA, Teixeira MA. Dilemmas and challenges for HIV prevention in representations of young Catholics. Online braz. j. nurs. [Internet]. 2018 [cited 2019 mar 14]; 17(1):97-108. DOI: https://doi.org/10.17665/1676-4285.20185909

14. Pontes APM, Oliveira DC, Gomes AMT. The principles of the Brazilian Unified Health System, studied based on similitude analysis. Rev. latinoam. enferm. [Internet]. 2014 [cited 2019 mar 14]; 22(1):59-67. DOI: http://dx.doi.org/10.1590/01041169.2925 .2395

15. Rodrigues AS, Oliveira JF, Suto CSS, Coutinho MPL, Paiva MS, Souza SS. Care for women involved with drugs: social representations of nurses. Rev. bras. enferm. [Internet]. 2017 [cited 2019 mar 14]; 70(1):71-8. DOI: http://dx.doi.org/10.1590/0034-7167-2016-0339

16. Wolter RP, SÁ CP. The relationship between representations and practices: the forgotten trail. Rev. Int. Cienc. Soc. Hum. [Internet]. 2013 [cited 2019 mar 14]; 23(1-2):87-105. DOI: https://doi.org/10.34117/bjdv6n7-126

17. Berman FD, Bourne PE. Let's make gender diversity in data science a priority right from the start. PLoS Biol. [Internet]. 2015 [cited 2019 mar 14]; 13(7):1-5. DOI: https://doi.org/10.1371/journal.pbio.1002206

18. Silva FCB, Brito RS. Pregnant women's perception concerning partners' absence during pre-natal care. Rev. RENE [Internet] 2010 [cited 2019 Mar 14]; 11(3):95-102. Available from: http://periodicos.ufc.br/rene/article/view/4594/3443

19. Okada MM, Hoga LAK, Borges ALV, Albuquerque RS, Belli MA. Domestic violence against pregnant women. Acta paul. enferm. [Internet]. 2015 [cited 2019 mar 14]; 28(3):270-4. DOI: http://dx.doi.org/10.1590/1982-0194201500045

20. Abate BA, Wossen BA, Degfie TT. Determinants of intimate partner violence during pregnancy among married women in Abay Chomen district, Western Ethiopia: a community based cross sectional study. BMC Women's Health [Internet]. 2016 [cited 2019 mar 14]; 16(1):1-8. DOI: http://dx.doi.org/10.1186/s12905-016-0294-6

21. Teixeira SVB, Moura MAV, Silva LR, Queiroz ABA, Souza QV, Netto LA. Intimate partner violence against pregnant women: the environment according to Levine's nursing theory. Rev. esc. enferm. USP [Internet]. 2015 [cited 2019 mar 14]; 49(6):882-9. DOI: http://dx.doi.org/10.1590/S0080-623420150000600002

22. Murray L, Dunne MP, Vo TV, Anh PNT, Khawaja NG, Cao TN. Postnatal depressive symptoms amongst women in Central Vietnam: a cross-sectional study investigating prevalence and associations with social, cultural and infant factors. BMC pregn. Childbirth [Internet]. 2015 [cited 2019 mar 14];15(3):1-12. DOI: http://doi.org/10.1186/s12884-015-0662-5

23. Araujo NB, Mandú ENT. Production of meanings among adolescents about self care during the pregnancy. Interface comun. saúde educ. [Internet]. 2016 [cited 2019 Mar 14]; 20(57):363-75. DOI: http://dx.doi.org/10.1590/1807-57622015.0301

24. Coutinho EC, Silva AL, Rodrigues SIM, Nelas PAB, Chaves CMB, Cabral LR et al. Social support during pregnancy and postpartum. Atas CIAIQ [Internet]. 2015 [cited 2019 mar 14]; 2(1):350-5. Available from: https://proceedings.ciaiq.org/index.php/CIAIQ/article/view/548/543

25. Souza AC, Figueiredo TAM, Del Priore M. Representações sociais sobre a mulher: um estudo com escolares. Rev. ECOS [Internet]. 2015 [cited 2019 Mar 14]; 10(1):25-31. Available from: https://periodicos.unemat.br/index.php/ecos/article/view/758/783 
26. Ferreira IS, Fernandes AFC, Lô KKR, Melo TP, Gomes AMF, Andrade IS. Perceptions of pregnant women about the role of partners in prenatal consultations. Rev. RENE [Internet]. 2016 [cited 2019 mar 14]; 17(3):318-23. DOI: https://doi.org/10.15253/2175-6783.2016000300003

27. Cabrita BAC, Silveira ES, Souza AC, Alves VH. The absence of the partner in prenatal care: challenges and achivements. R. pesq.: cuid. fundam. online [Internet]. 2012 [cited 2019 mar 14]; 4(3):2645-54. Available from: http://www.seer.unirio.br/index.php/cuidadofundamental/article/view/1851/pdf_605

28. Rodrigues C. Adam's rib: sexual differences from Levinas. Rev. Estud. Fem. [Internet]. 2011 [cited 2019 Mar 14]; 19(2):371-87. DOI: https://doi.org/10.1590/S0104-026X2011000200004

29. Ribeiro JP, Gomes GC, Silva BT, Cardoso LS, Silva PA, Strefling ISS. Participation of the rather during pregnancy, childbirth and puerperium: reflecting on the interfaces of nursing assistance. Espaç. saúde (Online). 2015 [cited 2019 mar 14]; 16(3):73-82. Available from: https://pesquisa.bvsalud.org/portal/resource/pt/lil-784095

30. King G, Lim JY, Kale AS, Lee LY. Adverse effects of young maternal age on neonatal outcomes. Singapore med. J. [Internet]. 2015 [cited 2019 mar 14]; 56(3):157-63. DOI: http://dx.doi.org/10.11622/smedj.2014194 